\title{
Artificial economic life: a simple model of a stockmarket
}

\author{
R.G. Palmer a,e, W. Brian Arthur b,e, John H. Holland ${ }^{\text {c,e }}$, Blake LeBaron ${ }^{\text {d,e }}$, \\ Paul Tayler ${ }^{\mathrm{f}}$ \\ a Department of Physics, Duke University, Durham, NC 27708-0305, USA \\ b Food Research Institute, Stanford University, Stanford, CA 94305-6084, USA \\ c Division of Computer Science \& Engineering, University of Michigan, Ann Arbor, MI 48103, USA \\ d Department of Economics, University of Wisconsin, Madison, WI 53706, USA \\ e Santa Fe Institute, 1660 Old Pecos Trail, Suite A, Santa Fe, NM 87501, USA \\ f Coopers \& Lybrand Deloitte MCS, Hillgate House, 26 Old Bailey, London EC4M 7PL, UK
}

\begin{abstract}
We describe a model of a stockmarket in which independent adaptive agents can buy and sell stock on a central market. The overall market behavior, such as the stock price time series, is an emergent property of the agents' behavior. This approach to modelling a market is contrasted with conventional rational expectations approaches. Our model does not necessarily converge to an equilibrium, and can show bubbles, crashes, and continued high trading volume.
\end{abstract}

\section{Approaches to economic theory}

In recent years the prevailing rational expectations approach to economic theory has been challenged from several quarters, and increasing interest has been shown in an alternative evolutionary economics viewpoint. In this paper we describe and contrast these paradigms, and discuss our artificial stockmarket model as an example of the evolutionary approach. Our approach is fundamentally based on the inductive theory of learning described in Arthur (1992) [1]. This stockmarket model may also be seen as a casestudy in artificial life; from a random soup of simple rules an intelligent system spontaneously organizes, and develops more and more sophisticated behavior as time goes on, rather like life emerging from a prebiotic soup.
We emphasize the background and general structure of our model, only indicating results in general terms. Related papers $[1,2]$ provide further details. The paper is written for physical scientists, without assuming any background in economics.

\subsection{Rational expectations theory}

In conventional economic theory the standard approach to most problems is fundamentally based on Rational Expectations (RE) theory. According to RE theory, agents deduce their optimum behavior by logical processes from the circumstances of any situation, assuming that other agents do likewise. Here an agent might be an individual, a firm, a state, etc. This seemingly reasonable approach in fact involves several 
strong (and unreasonable) assumptions, and has a number of undesired consequences. Nevertheless it has long been the regnant paradigm, perhaps in part because it leads to very appealing mathematics.

Among the assumptions normally made in RE theory are:

(i) Complete Information. All agents are assumed to have full knowledge of the problem.

(ii) Perfect Rationality. All agents are assumed to be perfectly able to deduce their optimum behavior, no matter how complex the computational problem.

(iii) Common Expectations. All agents are assumed to know that all others are working with the same information on the same "perfectly rational" basis. And they know that the others know this too, and that the others know that they know they know, and so on ad infinitum.

As a simple example, imagine 20 computer companies who are independently considering the adoption of a new standard $Z$ for a graphical user interface. A marketing analysis might show that all would benefit if at least 15 adopted $Z$, but that the adopters would experience a net loss if fewer than 15 adopted it. RE theory predicts that all companies will adopt $Z$ immediately, because they will all reason as follows. If I were the 15 th-20th company to consider adoption, it would obviously be in my interest to do so. If I were the 14 th, I would adopt because then it would be advantageous for the 15th-20th to do so. If I were the 13 th, I would do so because then the 14th would do so, by the preceding argument. As so on, all the way down to the first adopter. Since all will perform this reasoning, all will be ready to be first, and all will jump in immediately (and will expect the others to do so too). Note that the agents figure out the solution initially ("at time 0"), and that there is no subsequent dynamics, learning, or evolution.

Of course this outcome is not what would be expected in practice, partly because of the failure of the above assumptions, and partly because of other factors not included in our simple model. However it still serves to illustrate both the assumptions and the flavor of an RE argument, although in most applications the mathematical optimization problem is far more complicated.

In complex problems, RE theory runs into a number of difficulties, especially because the three assumptions listed above are typically not satisfied.

(i) Lack of Complete Information. Agents may have to learn about the context or about the other agents while the "game" is being played out. Problem contexts may themselves not be fully defined initially, only becoming explicit through the choices of the agents.

(ii) Lack of Perfect Rationality. Real persons and firms often aren't clever enough - or don't have enough computational power to compute a true optimum. And even if they have the power, they may not use it, preferring instead rules of thumb that have worked elsewhere.

(iii) Lack of Common Expectations. Different agents may well have different information about a situation, and may well use different approaches. They cannot rely on others to duplicate their own reasoning.

These difficulties lead in turn to predictions that do not always fit observed outcomes. And even when final outcomes are correctly predicted by RE theory, the theory is silent about the dynamical process (typically involving trial and error, and learning) that agents actually take to reach that solution.

\subsection{Evolutionary economics}

In part because of the difficulties with the standard RE theory, in recent years many researchers have investigated alternative approaches. Some have attempted to perturb away from the perfect rationality ideal with a variety of bounded rationality theories. These theories impose an inten- 
tional limitation on some aspect of an agent's task, such as the available knowledge, the computational time or complexity, the memory capacity, the forecasting repertoire, etc. One difficulty is that there are many dimensions in which to bound rationality, and no clear guiding principle for how to set the direction and distance from the zenith of perfect rationality.

Another approach, into which the current work falls, is to start from the opposite end of the scale with agents who initially have little rationality or specialized knowledge. The agents are then allowed to adapt, or learn, or evolve, eventually becoming reasonably expert in their own domains. There are a number of advantages to this approach, including

- None of the three assumptions discussed above for RE theory is required.

- Even the modeler does not need to have the knowledge or computational power to derive an optimum solution for each agent.

- The evolutionary approach is generally inductive, not deductive; the agents typically generalize patterns observed in the past to guide their behavior in the future. This inductive approach is much closer to normal human behavior than the deductive one of deriving particular choices from general principles $[1,8]$.

- The general approach is applicable even in situations where conventional RE theory produces no answers, e.g., due to lack of a single well-defined equilibrium solution.

- The approach can predict and interpret dynamical behavior, not just final outcomes.

- Agents can continue to adapt in a changing or ill-defined world (perhaps of their own making) whose characteristics cannot - or are not - known in advance.

The biggest disadvantages of the evolutionary approach are the general lack of analytic methods - most work is largely computational - and the plethora of possible algorithms for learning and adaptation. The field is presently in an exploratory phase, determining by explicit simulation the potentials and limitations of particu- lar evolutionary models. A narrowing of options and more rigorous results can be expected in the future.

\section{An artificial stockmarket}

\subsection{General framework}

Turning specifically to financial markets, we first construct the framework of a simple kind of stockmarket, and then consider different approaches (RE and evolutionary) to the agents' decision problem. Our market will have $S$ kinds of stocks labelled by $\alpha=1,2, \ldots, S$, and $N$ agents labelled by $i=1,2, \ldots, N$. The agents are not necessarily homogeneous; they may have quite different operating principles. For simplicity we make time $t$ discrete, so $t=0,1,2, \ldots$, and refer to the interval from $t-1$ to $t$ as the $t$ th period. There is no predefined time horizon; in principle the market continues for ever.

At each time $t$, each agent $i$ has some number of shares (or holding) $h_{i}^{\alpha}(t)$ of each stock $\alpha$. There are no complex instruments such as options, and no direct interaction between pairs of agents. The agent's essential problem is to choose $h_{i}^{\alpha}(t)$ at each time $t$, given various constraints such as a finite net wealth. The goal might be to maximize expected (mean) profit, or might involve a more complicated "utility function" which takes risk into account. The price $p^{\alpha}(t)$ per share of each stock depends mainly on the overall buying and selling behavior of the agents. The companies issuing the stocks may also pay cash dividends $d^{\alpha}(t)$ per share to each stockholder, in an amount depending on company success and policies. Agents can thus make profit in two ways, through the dividend stream and through speculation, relying on price changes of their shares.

In addition to stock holdings we need to take into account other assets of each agent, so that not all wealth needs to be invested in stock. For simplicity we regard all other assets collectively 
as cash, or money $M_{i}(t)$. An agent's total wealth $w_{i}(t)$ at any time $t$ is thus given by

$$
w_{i}(t)=M_{i}(t)+\sum_{\alpha} h_{i}^{\alpha}(t) p^{\alpha}(t)
$$

During the $t$ th period, the price per share of stock $\alpha$ changes from $p^{\alpha}(t-1)$ to $p^{\alpha}(t)$ and a dividend $d^{\alpha}(t)$ is declared. We also assume that the agent's cash is invested in a fixed-rate fund such as a savings account, which pays an interest rate $r$ per period so that $M_{i}(t-1)$ becomes $(1+r) M_{i}(t-1)$. Accounting for these changes, the agent's wealth at the end of a period is given by

$$
\begin{aligned}
& w_{i}(t)=(1+r) M_{i}(t-1) \\
& \quad+\sum_{\alpha} h_{i}^{\alpha}(t-1)\left[p^{\alpha}(t)+d^{\alpha}(t)\right],
\end{aligned}
$$

which is a net change of

$$
\begin{aligned}
& \Delta w_{i}(t) \equiv w_{i}(t)-w_{i}(t-1)=r M_{i}(t-1) \\
& \quad+\sum_{\alpha} h_{i}^{\alpha}(t-1)\left[p^{\alpha}(t)+d^{\alpha}(t)-p^{\alpha}(t-1)\right]
\end{aligned}
$$

from the beginning of that period.

Before the next period begins the agents have an opportunity to change their holdings, choosing $h_{i}^{\alpha}(t)$ and $M_{i}(t)$ subject to the constraints:

(i) Fixed total wealth: Eq. (1) must apply at every $t$. Given the new wealth $w_{i}(t)$ from Eq. (2), this budget constraint is just a linear condition on $M_{i}(t)$ and the $h_{i}^{\alpha}(t)$ 's.

(ii) Positivity: $M_{i}(t) \geq 0$, and $h_{i}^{\alpha}(t) \geq 0$ for all $\alpha$. Actually these constraints can be relaxed to allow borrowing (possibly with a larger value of $r$ when $M_{i}(t)$ is negative) or selling short (negative holdings), but we still need lower bounds on $M_{i}(t)$ and $h_{i}^{\alpha}(t)$ for stability.

(iii) Market clearing conditions: Individual agents may not be able to achieve the stock holdings they desire, because for every seller there must be a buyer, and viceversa; the total number of shares of each stock is fixed:

$$
\sum_{i} h_{i}^{\alpha}(t)=H^{\alpha} \quad \forall t .
$$

There are a number of ways to implement market clearing conditions, but here we only describe the simplest in detail. For each stock, each agent can submit either a bid to buy $b_{i}^{\alpha}(t)$ shares, or an offer to sell $o_{i}^{\alpha}(t)$ shares - in both cases at the current price $p^{\alpha}(t)$ - or neither. We define $b_{i}^{\alpha}(t)=0$ and/or $o_{i}^{\alpha}(t)=0$ for the remaining cases. Bids and offers need not be integers; the stock is infinitely divisible. Then

$B^{\alpha}(t)=\sum_{i=1}^{N} b_{i}^{\alpha}(t)$,

$O^{\alpha}(t)=\sum_{i=1}^{N} o_{i}^{\alpha}(t)$

are the totals of the bids and offers for stock $\alpha$ at time $t$. If $B^{\alpha}(t)=O^{\alpha}(t)$, then all bids and all offers are fully satisfied, giving

$h_{i}^{\alpha}(t)=h_{i}^{\alpha}(t-1)+b_{i}^{\alpha}(t)-o_{i}^{\alpha}(t)$

(where either $b_{i}^{\alpha}(t)$ or $o_{i}^{\alpha}(t)$ is zero for each $\alpha)$. If, however, $B^{\alpha}(t)>O^{\alpha}(t)$, then all offers are fully satisfied, and a fraction $O^{\alpha}(t) / B^{\alpha}(t)$ of each bid is filled, giving

$h_{i}^{\alpha}(t)=h_{i}^{\alpha}(t-1)+\frac{O^{\alpha}(t)}{B^{\alpha}(t)} b_{i}^{\alpha}(t)-o_{i}^{\alpha}(t)$.

Similarly if $B^{\alpha}(t)<O^{\alpha}(t)$, then all bids are fully satisfied, and a fraction $B^{\alpha}(t) / O^{\alpha}(t)$ of each offer is filled, giving

$$
h_{i}^{\alpha}(t)=h_{i}^{\alpha}(t-1)+b_{i}^{\alpha}(t)-\frac{B^{\alpha}(t)}{O^{\alpha}(t)} o_{i}^{\alpha}(t) .
$$

All these cases can be subsumed into

$$
\begin{aligned}
h_{i}^{\alpha}(t)= & h_{i}^{\alpha}(t-1)+\frac{V^{\alpha}(t)}{B^{\alpha}(t)} b_{i}^{\alpha}(t) \\
& -\frac{V^{\alpha}(t)}{O^{\alpha}(t)} o_{i}^{\alpha}(t),
\end{aligned}
$$

where $V^{\alpha}(t) \equiv \min \left(B^{\alpha}(t), O^{\alpha}(t)\right)$ is the number of trades (or volume) in stock $\alpha$. 
This rationing scheme is far from satisfactory in general, both because of lack of realism, and because it can lead to violations of the positivity constraints. For example, an agent could plan to sell a large amount of stock $A$ to raise the funds to buy $B$, but end up with $M_{i}(t)<0$ because the sale of $A$ was rationed while the purchase of $B$ was not. A better scheme is to relax the constraint (4), allowing temporary imbalances between stock purchases and sales to be made up by changes in the stock inventory of a market specialist. The specialist - an actual person in many real markets - has to control the price so that his or her inventory stays within acceptable bounds. Another more elaborate possibility is to have the agents engage in an iterative auction-like process to buy or sell stock, adjusting the price until supply $O^{\alpha}(t)$ and demand $B^{\alpha}(t)$ are equal. We will report on these and other options elsewhere [2]; here we use only the simple rationing scheme, and will ultimately avoid the positivity problem by limiting ourselves to a single stock.

To complete our specification of the market, we need to detail how the dividends $d^{\alpha}(t)$ and prices $p^{\alpha}(t)$ are fixed. For the dividends we choose a purely stochastic process, entirely independent of the agents' actions. In a sense the dividend stream is a noise source, somewhat like a physical temperature, that drives the market. We have explored a number of different random processes for $d^{\alpha}(t)$ including: simple random number generators without any $t \rightarrow t+1$ correlation; regular ramps and square waves (to see if our agents can learn simple periodicities); and various Markov processes in which $d^{\alpha}(t)$ depends on $d^{\alpha}(t-1)$. The simplest case having any claim to realism is a discrete colored noise process (or, equivalently, an Ornstein-Uhlenbeck process, or an AR (1) process) for the logarithm of $d^{\alpha}(t) / \bar{d}^{\alpha}$ (where $\bar{d}^{\alpha}$ simply sets the scale):

$\log \frac{d^{\alpha}(t)}{d^{\alpha}}=a_{\alpha} \log \frac{d^{\alpha}(t-1)}{d^{\alpha}}+b_{\alpha} \xi^{\alpha}(t)$.

Here $\xi^{\alpha}(t)$ is a Gaussian noise source with mean 0 and variance $\sigma_{\alpha}^{2}$, and $a_{\alpha}$ and $b_{\alpha}$ are positive parameters such that $a_{\alpha}^{2}+b_{\alpha}^{2}=1$. It is easy to show that $\log d^{\alpha}(t) / \bar{d}^{\alpha}$ has mean 0 , variance $\sigma_{\alpha}^{2}$, and an exponentially decaying autocorrelation function with correlation time $\tau_{\alpha}=1 / \log a_{\alpha}$.

The prices $p^{\alpha}(t)$ must depend on the bids and offers of the agents. The price of a stock should rise if the demand for it exceeds the supply (more agents wanting to buy than to sell), and fall if the supply exceeds the demand. Choice of a detailed mechanism is interrelated to the way bids and offers are matched; an iterative auction process, for example, would inherently determine prices itself. For the rationing scheme chosen here we use a very simple price adjustment scheme, based solely on the excess demand $B^{\alpha}(t)-O^{\alpha}(t)$ :

$p^{\alpha}(t+1)=p^{\alpha}(t) \cdot\left\{1+\eta\left[B^{\alpha}(t)-O^{\alpha}(t)\right]\right\}$.

The parameter $\eta$ is a crucial determinant of the ultimate behavior; small $\eta$ leads to very slow adjustment of prices, while large $\eta$ gives large oscillations. In most cases we have instituted an adaptive mechanism for $\eta$ itself, based on feedback from the number of recent reversals in direction of the price trend, and aiming to keep the response near to critical damping. But here we will keep $\eta$ fixed, and small enough so that $\eta\left[B^{\alpha}(t)-O^{\alpha}(t)\right] \ll 1$.

This completes our specification of the market itself. All that is undetermined is the way in which individual agents choose their bids $b_{i}^{\alpha}(t)$ or offers $o_{i}^{\alpha}(t)$, based on the information available to them. We assume that that information consists of the entire past history of the market, including prices $p^{\alpha}\left(t^{\prime}\right)$ and dividends $d^{\alpha}\left(t^{\prime}\right)$ for $t^{\prime} \leq t$, and total bids $B^{\alpha}\left(t^{\prime}\right)$ and offers $O^{\alpha}\left(t^{\prime}\right)$ for $t^{\prime}<t$. We also sometimes introduce a purely random sunspot variable $\psi(t)$. Although $\psi(t)$ is not causally connected to the market, the prices can nevertheless become correlated with $\psi(t)$ if agents "believe" that it has predictive power and coordinate their actions around its fluctuations. 


\subsection{Rational expectations approach}

A simple ("risk free") RE approach to the market just described would be based on a behavioral equation of the form $[1,11]$

$p^{\alpha}(t)=\beta E\left[p^{\alpha}(t+1)+d^{\alpha}(t+1) \mid I(t)\right]$,

where $\beta=1 /(1+r)$, and $E[\cdot \mid I(t)]$ means an expected value (prediction) given all the information $I(t)$ available at the current time $t$. We assume the efficient market hypothesis that the price reflects (i.e., values appropriately) all accessible information about the future. Eq. (13) says that the current price $p^{\alpha}(t)$ per share of stock $\alpha$ should reflect the best estimate of its value $p^{\alpha}(t+1)+d^{\alpha}(t+1)$ at the end of the period, discounted by the factor $\beta$ to allow for the increase in the value of money implied by the interest rate $r$. The reasoning behind Eq. (13) is that any other value for $p^{\alpha}(t)$ would represent an opportunity to make a profit, which rational agents would take (if they care nothing about risk). If, for example, the actual price of a stock were lower than given by Eq. (13), then many agents would attempt to invest in it, thus driving the price up until Eq. (13) was satisfied. This is called arbitrage.

If we iterate Eq. (13), using the law of iterated expectations $E[E[x \mid I(t+1)] \mid I(t)]=$ $E[x \mid I(t)]$, we obtain

$p^{\alpha}(t)=\sum_{n=1}^{\infty} \beta^{n} E\left[d^{\alpha}(t+n) \mid I(t)\right]$,

so that the price today should just depend on an appropriately discounted series of expected future dividends. If the dividend series had a constant expected value, so that $E\left[d^{\alpha}(t+n) \mid I(t)\right]=\bar{d}^{\alpha} \forall n$, this would reduce to

$p^{\alpha}(t)=d^{\alpha} / r$

which gives the fundamental value of stock $\alpha$ in this approximation. Note, however, that a col- ored noise process such as Eq. (11) does not satisfy this constant expected value assumption.

More sophisticated RE approaches are possible. In particular we could allow for risk aversion in the agents. Typically this would lower the price $p^{\alpha}(t)$ from that given by Eq. (13) or Eq. (15) by an amount proportional to the variance of the prediction for $p^{\alpha}(t+1)+d^{\alpha}(t+1)$; riskier returns are worth less. But the essential flavor of the approach is not changed by such improvements.

The RE approach implicitly assumes that all agents compute the same expectation values $E\left[p^{\alpha}(t+1)+d^{\alpha}(t+1) \mid I(t)\right]$ for each stock; otherwise the arbitrage argument fails. This assumes not only that they all have the same information $I(t)$, but also that they form expectations in the same way, and indeed know that others will do so too. In practice these assumptions will fail; no two agents are likely to have exactly the same information, and agree that there is a unique objective way to compute the required expectation value, and know what that unique method is. Moreover, individual agents cannot form their expectations in a fully rational way unless they know how others form theirs, so all are reduced to subjective beliefs about each other's behavior. In Keynes' words [9], thcy must "devote [their] intelligences to anticipating what average opinion expects the average opinion to be."

These fundamental difficulties of the RE approach lead in turn to predictions that do not correspond to the empirical behavior of real markets [4]. In particular the RE theory predicts low trading volume; there is no reason for agent $A$ to sell shares to agent $B$ if they both have the same information and expectations. Further, there is no room for market bubbles or crashes, or any sort of market psychology or moods. Finally, it should not be possible to make any profit by technical trading - attempting to predict future stock prices by recognizing and exploiting patterns in past prices - since any such opportunities should be removed by 
arbitrage; this is a direct consequence of the efficient market hypothesis.

In real markets there is much higher trading volume than RE predicts, there are bubbles, crashes, and moods, and many traders secm to live by technical trading. These and other anomalies can be reconciled with the RE approach only by extending or modifying it, for instance by introducing heterogeneous expectations [6], or by allowing Bayesian learning of parameters [3]. However none of these approaches is entirely satisfactory, and none provides a truly dynamical picture of the market.

\subsection{Evolutionary approach}

Instead of the RE approach, we propose an inductive model in which we start with agents who have little knowledge or reasoning ability. The agents trade on the basis of internal models or rules that are initially very poor. By observing the success or failure of these internal models the agents can improve them, select among alternatives, and even generate totally new rules. Thus over time their behavior becomes more sophisticated, and they become able to recognize and exploit many patterns in the market. The stock prices $p^{\alpha}(t)$ themselves reflect the aggregate behavior of the agents; phenomena like bubbles, crashes, and market moods can emerge as collective phenomena. Because they both create and exploit the prices series, the agents are essentially coevolving, even though they do not interact directly with one another.

The inductive approach provides a dynamical picture of a market and avoids most of the previously discussed problems of RE theory. It is also inherently closer to the way humans typically make decisions in complex situations [8]. They start by making mental models or hypotheses, based on past experience and training. These models may directly imply a course of action, or they may let them anticipate the outcome of various possible actions, on which basis a choice can be made. In any case, humans also observe their own successes and failures, learning to modify or abandon unsuccessful mental models, and to rely more strongly on successful ones.

Our present approach is computational. We definc a framcwork for the agents' behavior in which learning and adaptation is possible, and then we run simulations of a whole market to see how both the agents and the market behave. Some analysis and interpretation is certainly possible, but no detailed analytic theory is yet to hand. There are some rigorous results for simpler systems of adaptive economic agents $[10,12]$, and of course there is much work on learning systems in general, but none of this is directly applicable to entire markets. Thus an exploratory computational approach seems appropriate.

\subsection{Condition-action agents}

To implement an inductive approach we must specify in detail how agents choose their bids or offers. We have been experimenting with a number of approaches, including agents who explicitly forecast the future and perform a riskaversion computation to choose their optimum holdings [2]. But here we describe only a simpler class of agents, based on a classifier system and a genetic algorithm [5,7].

Before proceeding, we specialize to a single stock, and drop the $\alpha$ superscripts. Most phenomena of interest are already present with a single stock, and the restriction removes the difficulty with the positivity condition. We now reduce the bid/offer decision to a simple ternary choice:

(i) Bid to buy one share: $b_{i}(t)=1, o_{i}(t)=0$.

(ii) Offer to sell one share: $o_{i}(t)=1, b_{i}(t)=0$. (iii) Neither: $b_{i}(t)=o_{i}(t)=0$.

Provided one period (from $t$ to $t+1$ ) represents a short interval in terms of the dividend autocorrelation time $\tau$ and the price adjustment timescale (set by $\eta$ ), the restriction of demand to \pm 1 is not serious; larger changes can be achieved by a sequence of smaller ones. 
In the spirit of a classifier system, each agent has many condition-action rules. We typically use $R=60$ rules per agent, labelling rules by $k=$ $1,2, \ldots, R$. Each agent $i$ has its own set of rules, independent of all other agents, so there are $N R$ rules in all, labelled by $(i, k)$. Each of these rules has three components:

(i) A condition part, that governs when (under what market conditions) the rule is activated. We discuss this further in a moment.

(ii) An action $a_{i k}= \pm 1$, representing either buy $(+1$, bid one share $)$ or sell $(-1$, offer one share).

(iii) A strength $s_{i k}(t)$, representing how successful the rule has been at suggesting wealth-increasing actions in the past.

Each time that the agent has to make a decision it first lists those of its rules that are activated and have $s_{i k}(t)>0$. Next it selects one of these randomly, with probability proportional to strength. The action of this selected rule then gives the agent's decision: buy or sell. If the list is empty, then the agent makes neither a bid nor an offer.

The strengths of all activated rules (not just selected ones) are updated at the end of the period according to:

$$
\begin{aligned}
& s_{i k}(t)=(1-c) s_{i k}(t-1)+ \\
& c a_{i k}[p(t)-(1+r) p(t-1)+d(t)] .
\end{aligned}
$$

The term in square brackets represents the net profit made by investing in one share of stock for the past period, rather than leaving the money in the bank; compare Eq. (3). $c$ is a small parameter (e.g., 0.01$)$ so that $s_{i k}(t)$ accumulates over a long period an exponentially-weighted moving average of the net profit potential of the rule's action under circumstances in which it is activated. To avoid occasional problems we restrict strengths to $s_{\min } \leq s_{i k}(t) \leq s_{\max }$.

Note that any rule can be injected into the population (with initial strength 0 , say) without negatively affecting the agent's behavior. If the rule is poor it will sink to negative strength and never be selected. If it is good it will gain strength and may come to be used as a basis for a decision. For example, there could well be two rules with identical conditions but opposite actions, but only one would come to have positive strength.

The condition part of each rule consists of a string of symbols such as $* * * 1 * 0 * * * 11 * *$, drawn from the ternary alphabet $\{0,1, *\}$. These strings are matched against a single binary string (with 0 and 1 symbols only) that represents the current state of the market. 0 's and 1's in the condition string only match 0 's and 1's respectively in the market string, whereas *'s are don't care symbols that match either 0 or 1 . Thus, for example, the above condition string matches a market state of 0101001101101 but not 1001011100101 .

The number and meaning of the bits in the market state string can be adjusted to give the agents more or less information. We typically use strings of length 70-80 symbols, providing a mixture of short-term and long-term information, such as:

- The price is above 1.2 times fundamental value (as given by Eq. 15).

- The dividend went up two periods ago.

- The 100-period moving average of price went up (compared to the previous 100 periods).

- The 20-period moving average of price is above the 100-period moving average.

In each case the appropriate bit is 1 if the corresponding statement is true, 0 if it is false.

The structure described so far is a simple classifier system; the rules classify the states of the environment (market state) into many categories, depending on which are activated, and then provide probabilities for each possible action to be taken in each category. By itself it simply assigns strengths to a pre-defined set of rules. But this is easily extended by adding a genetic algorithm which generates new rules, so that the population of rules can evolve towards ever better ones. Our genetic algorithm is applied at random times (in a Poisson process) to 
each agent, and has the effect of replacing 10$20 \%$ of its rules by new ones. It selects some of the weakest rules for replacement, and initially makes copies (clones) of some of the strongest rules to replace them, selecting candidates with probability proportional to their strength. The clones may then be modified by random mutation and crossover. Mutation means that a few symbols are randomly changed, with probabilities adjusted so that the average number of don't care symbols stays constant. Crossover means that a pair of "parent" strings is selected (from among the clones) and used to generate two "offspring" strings, each of which gets its symbols partly from one parent and partly from the other; the idca is to combine good building blocks (substrings) present in the two parents [5].

We also apply two further operations to the whole new population. Firstly, rules that are particularly weak (negative strength) have their actions reversed; if buying was bad, then selling should be good. Secondly, rules that have not been activated in a long time are generalized, changing some of their specific $0 / 1$ symbols to *'s.

\section{Results and conclusions}

As stated initially, this paper describes mainly our rationale and overall design. Quantitative results will be presented elsewhere [2]. Qualitatively our main observations are as follows.

(i) In sufficiently simple cases - with few agents, or few rules per agent, or a lowvariance dividend stream - the agents converge to an equilibrium in which price tracks fundamental value (Eq. 15), volume stays low, and there are no appreciable anomalies such as bubbles or crashes. The agents become relatively homogeneous, relying mainly on simple rules such as buy when the price is below fundamental value. The overall behavior is just what would be expected from RE theory and the efficient market hypothesis.

(ii) On the other hand, in a richer environment, there is no evidence of equilibrium. Instead we obtain what we call economic life; as described in the following observations, there is rich evolving behavior that becomes more complex over time.

(iii) Although the price frequently stays close to fundamental value, it also displays major upward and downward deviations which may be called bubbles and crashes. Often these have no simple explanation; the effect is collective, and cannot always be traced to a simple rule or instability. It is reasonable to think of them as corresponding to moods of the market. But one mechanism is clear; a set of condition-action rules can be collectively self-fulfilling and hence give positive feedback that amplifies any small fluctuatation from equilibrium. For example, a trend-following rule that simply suggests buying stock when the price is rising will, once triggered, create demand that drives the price up further.

(iv) The agents become quite heterogeneous, using very different rules.

(v) Trading volume varies greatly, and is sometimes quite high. This reflects the heterogeneity of the agents - a set of identical agents would never want to buy from one another. There tend to be long periods of relative calm, interspersed with episodes of high-volume activity. These episodes do not always coincide with the bubbles and crashes.

(vi) Over time, the complexity of the agents increases steadily, even though the marketlevel phenomena appear relatively stationary. One measure of agent complexity is the average number of non-* symbols in their rules. A steady increase in this measure is fairly common in classifier systems, and might just reflect a random increase in the use of redundant bits, or the details 
of the genetic algorithm. But more likely it reflects the discovery of addenda and exceptions to gross responses uncovered early on, leading to a default hierarchy of rules [8].

(vii) If a trained agent is extracted from the market and then reinserted much later, it tends to do rather poorly. The rules needed for success change in time - there is no stationary optimum strategy.

(viii) The ecology of agents can adapt to new situations such as a changed dividend stream. They never get locked into a particular approach, but automatically choose a balance between exploration of new rules and exploitation of old successful ones.

(ix) An initially uniform wealth distribution evolves into a wide distribution, with some agents becoming much more wealthy than others over long periods. This reflects agent heterogeneity and "luck"; the chance discovery of good rules can make certain agents very rich for a while. However over very long periods the identity of the winners and losers changes, although the statistical distribution remains approximately constant.

It is worth noting explicitly the artificial life aspects of our model. We find the self-formation of an autonomous economy that bootstraps itself up from randomized "stupid" behavior to organized mutually-adapted behavior. From a random soup of simple rules, an "intelligent" system spontaneously organizes. Thus we have modelled the origins of economic life among interacting agents in the same sense that others model the origin of biological life among organic molecules. What distinguishes this from standard equation-based equilibrium economics is the ability of the agents to learn, and of the system to bootstrap itself to a high order of mutual behavior, rather than merely to implement some simple optimizing rule at an equilibrium.

In all, our models of a stockmarket can reproduce the major features (as well as some de- tailed statistics, to be discussed elsewhere [2]) of real markets, including dynamical and nonequilibrium phenomena. It does not require and indeed rejects - the restrictive assumptions of rational expectations theory. Its relative disadvantage is that it is largely a computational model, without immediate prospects for rigorous mathematical results, whereas rational expectations theory leads to rich mathematical formalism.

We see our stockmarket model, and others of its class, as a fertile testbed for exploring markets, adaptive agents, and a class (distinguished especially by the lack of direct agent-agent interactions) of artificial life. For example, we can explore the effect of changing the market mechanism, for instance by adding a specialist with inventory, or by imposing transaction costs or price controls, and see how the market efficiency is affected. We can investigate what mechanisms would be effective for stopping or limiting bubbles and crashes. We can analyze various computerized trading schemes and evaluate their effect on the stability of the market. We can explore the difference between homogeneous and heterogeneous traders, and see the effect of adding naive "noise" traders who are not principally motivated by profit (and can therefore be exploited by others).

We can also investigate many variations at the agent level, including different learning techniques and different goals (including risk aversion). We can inquire into the effect of giving certain agents inside information. We can try limiting the computational ability of some agents. We can set up a framework in which agents can go bankrupt and be replaced by clones of better agents, so that the whole population of agents evolves.

Some of these projects are already under way; others are on the distant horizon. We also expect to make public our software (which includes dynamical displays of market and agent behavior) within the next year, so that others can share in the endeavor. 


\section{Acknowledgement}

This reasearch was supported in part by grants to the Santa Fe Institute, including core funding from the John D. and Catherine T. MacArthur Foundation, the National Science Foundation (PHY-8714918), The Alex Walker Educational and Charitable Foundation, and the U.S. Department of Energy (ER-FG05-88ER25054).

\section{References}

[1] W. Brian Arthur, On learning and adaptation in the economy, Sante Fe Institute Working Paper 92-07038 (1992).

[2] W. Brian Arthur, John H. Holland, Blake LeBaron, R.G. Palmer and Paul Tayler, An evolutionary model of a stockmarket (1994), in preparation.

[3] I. Blume and D. Easley, Evolution and market behavior, J. Econ. Theory 58 (1992) 9-40.

[4] M.E. Blume and J.J. Siegel, The theory of security pricing and market structure, Financial Markets, Institutions and Instruments 1, No. 3 (1992).

[5] David E. Goldberg, Genetic Algorithms in Search, Optimization and Machine Learning (AddisonWesley, Reading, MA, 1989).

[6] S.J. Grossman, On the efficiency of competitive stock markets where traders have diverse information, J. Finance 31 (1976) 573-585.

[7] J.H. Holland, Adaptation in Natural and Artificial Systems (MIT Press, Cambridge, MA, 1992).

[8] J.H. Holland, K.J. Holyoak, R.E. Nisbett and P.R. Thagard, Induction (MIT Press, Cambridge, MA, 1986).

[9] J.M. Keynes, The General Theory of Employment, Interest and Money (Macmillan, London, 1936).

[10] M. Lettau and H. Uhlig, How Well Do Artificially Intelligent Agents Eat Cake?, Princeton University manuscript (1993).

[11] R.E. Lucas, Asset prices in an exchange economy, Econometrica 46 (1978) 1429-1445.

[12] R. Marimon, E. McGrattan and T.J. Sargent, Money as a medium of exchange in an economy with artificially intelligent agents, J. Econ. Dynamics \& Control 14 (1990) 329-373. 